Research paper

\title{
Towards the bioequivalence of pressurised metered dose inhalers 2 . Aerodynamically equivalent particles (with and without glycerol) exhibit different biopharmaceutical profiles in vitro
}

\author{
M. Haghi ${ }^{\mathrm{a}, \mathrm{b}}$, M. Bebawy ${ }^{\mathrm{c}}$, P. Colombo $^{\mathrm{d}}$, B. Forbes ${ }^{\mathrm{e}}$, D.A. Lewis ${ }^{\mathrm{f}}$, R. Salama ${ }^{\mathrm{a}, \mathrm{b}}$, D. Traini ${ }^{\mathrm{a}, \mathrm{b}}$, P.M. Young ${ }^{\mathrm{a}, \mathrm{b}, *}$ \\ ${ }^{a}$ Respiratory Technology, The Woolcock Institute of Medical Research, The University of Sydney, Australia \\ ${ }^{\mathrm{b}}$ Discipline of Pharmacology, Faculty Medicine, The University of Sydney, Australia \\ ${ }^{\mathrm{C}}$ Graduate School of Pharmacy Health-Pharmacy, University of Technology Sydney, Sydney, Australia \\ ${ }^{\mathrm{d}}$ Department of Pharmacy, University of Parma, Parma, Italy \\ e Institute of Pharmaceutical Science, King's College London, London, UK \\ ${ }^{\mathrm{f}}$ Chiesi Limited, Chippenham, UK
}

\section{A R T I C L E I N F O}

\section{Article history:}

Available online 22 March 2013

\section{Keywords:}

pMDI

Beclomethasone dipropionate

Bioequivalence

Non-volatile excipients

Glycerol

Epithelial cell model

Calu-3

\begin{abstract}
A B S T R A C T
Two solution-based pressurised metered dose inhaler (pMDI) formulations were prepared such that they delivered aerosols with identical mass median aerodynamic diameters, but contained either beclomethasone dipropionate (BDP) alone (glycerol-free formulation) or BDP and glycerol in a 1:1 mass ratio (glycerol-containing formulation). The two formulations were deposited onto Calu-3 respiratory epithelial cell layers cultured at an air interface. Equivalent drug mass ( $\sim 1000 \mathrm{ng}$ or $\sim 2000 \mathrm{ng}$ of the formulation) or equivalent particle number (1000 ng of BDP in the glycerol-containing versus $2000 \mathrm{ng}$ of BDP in the glycerol-free formulation) were deposited as aerosolised particles on the air interfaced surface of the cell layers. The transfer rate of BDP across the cell layer after deposition of the glycerol-free particles was proportional to the mass deposited. In comparison, the transfer of BDP from the glycerol-containing formulation was independent of the mass deposited, suggesting that the release of BDP is modified in the presence of glycerol. The rate of BDP transfer (and the extent of metabolism) over $2 \mathrm{~h}$ was faster when delivered in glycerol-free particles, $465.01 \mathrm{ng} \pm 95.12 \mathrm{ng}$ of the total drug $(20.99 \pm 4.29 \%$; BDP plus active metabolite) transported across the cell layer, compared to $116.17 \mathrm{ng} \pm 3.07 \mathrm{ng}(6.07 \pm 0.16 \%)$ when the equivalent mass of BDP was deposited in glycerol-containing particles. These observations suggest that the presence of glycerol in the maturated aerosol particles may influence the disposition of BDP in the lungs.
\end{abstract}

(c) 2013 Elsevier B.V. All rights reserved.

\section{Introduction}

The efficacy of inhaled corticosteroids for the treatment of asthma is well documented, with national and international treatment guidelines recommending their use for control of mild persistent, moderate and severe asthma [1,2]. Beclomethasone dipropionate (BDP) is one of the most commonly used corticosteroids in the treatment of asthma and, after deposition in the lung, undergoes rapid metabolism via esterase enzymes to the active metabolite, beclomethasone-17-monopropionate (BMP) [3]. Several devices have been developed to deliver BDP to the pulmonary system including nebulisers, dry powder inhalers and pressurised metered dose inhalers (pMDI). pMDIs are the most common devices for

* Corresponding author. Respiratory Technology, The Woolcock Institute of Medical Research, The University of Sydney, NSW 2006, Australia. Tel.: +61 2 9114 0350; fax: +61291140014

E-mail address: paul.young@sydney.edu.au (P.M. Young). delivery of BDP with marketed products including solution-based inhalers, e.g. QVAR ${ }^{\circledR}$ by $3 \mathrm{M}$ Ltd. and Clenil ${ }^{\circledR}$ by Chesi Ltd. (also licenced as Sanasthmax ${ }^{\circledR}$ and Becloforte ${ }^{\circledR}$ ) and suspension inhalers, e.g. Becotide ${ }^{\circledR}$ by GSK Ltd.

Historically, BDP pMDIs were formulated in chlorofluorocarbon (CFC) propellant; however, with the regulatory phase out of CFCs, reformulation in more environmental friendly hydrofluoroalkanes (i.e. HFA 134a and 227) was undertaken. During the CFC-HFA transition, different reformulation approaches were explored resulting a plethora of patents within the field [4]. For example, BDP was formulated as an ultrafine inhalation aerosol by solubilising the drug in an ethanol-HFA 134a-based system [5]. Similarly, Modulite ${ }^{\mathrm{TM}}$ technology utilised an ethanol-HFA-based solution, but additionally incorporated non-volatile components, such as glycerol, to generate a larger mass median aerodynamic diameter (MMAD), equivalent to that of the original CFC-based systems [6]. While the former approach required extensive clinical trials due to different regional lung deposition [7], the Modulite technology was 
tuned to have a similar deposition profiles to that of the former CFC formulations.

Several clinical studies have evaluated the ultrafine and Modulite products and compared these to suspension CFC formulations in terms of pharmacokinetics (systemic exposure) and clinical efficacy [3]. The ultrafine BDP formulations with a mass median aerodynamic diameter (MMAD) ca. $1.1 \mu \mathrm{m}$ resulted in greater systemic uptake and area under the curve (AUC) blood plasma concentrations compared to the larger Modulite (MMAD ca. $2.6 \mu \mathrm{m}$ ) or CFC formulations (MMAD ca. $4 \mu \mathrm{m}$ ) [3]. The area under the plasma concentration curve for the BMP in the Modulite formulations was reported to be markedly different than QVAR, but not dissimilar to the CFC formulations even though the MMAD's were different [8]. In addition, the Modulite formulations were shown to have similar clinical outcomes to CFC formulations in terms of Forced Expiratory Volume and morning serum cortisol concentrations [9]. A recent review by Derom and Pauwels has summarised clinical studies utilising BDP in different HFA formulations [3]; however, while these studies provide an insight into the pharmacokinetics and dynamics of HFA BDP systems, direct comparison between two formulations are difficult due to the nature of the study designs.

Interestingly, ex vivo and in vitro studies comparing ultrafine and Modulite-based pMDIs have shown significant differences in drug disposition $[10,11]$. A study by Freiwald et al., employing an extracorporally ventilated and re-perfused human lung lobe, showed that Modulite pMDI formulations had a significantly slower tissue penetration rate that could be attributed to the particle topology and dissolution rate [11]. A subsequent study by Grainger et al. produced similar findings using an in vitro Calu-3 epithelial cell line-based respiratory drug absorption model and a Franz diffusion cell method to measure dissolution [10]. Particles generated from QVAR $^{\circledR}$ (containing HFA, BDP and ethanol) and Sanasthmax ${ }^{\circledR}$ (containing HFA, BDP, ethanol and glycerol as a particle performance modifier) products were compared in terms of their dissolution and transport across an air interface Calu- 3 cell line after aerosol deposition. The particles generated by the QVAR formulation dissolved more rapidly and resulted in more rapid transport across the epithelial cell layer; however, this difference could not be attributed to a single particle property, since the particle size $($ MMAD QVAR $=1.1 \mu \mathrm{m}$; Sanasthmax $=2.8 \mu \mathrm{m})$ crystallinity and non-volatile excipients (i.e. only Sanasthmax contained glycerol) varied [10].

Current approved approaches to assess bioequivalency of oral inhaled products include the following: unit dose sampling, cascade impaction, spray pattern and plume geometry. There are, however, general issues regarding the in vitro tests [12]. Cascade impaction techniques are useful for general understanding of lung deposition; however, the results are not well correlated with regional lung deposition and the fate of particles deposited on the airway epithelium, the fist barrier encountered by microparticles after inhalation.

In order to study how individual inhaler formulation variables affect the biopharmaceutical performance of the particles generated, it is important that each variable is studied in isolation. The presence of glycerol as a non-volatile additive in the formulation is interesting since glycerol is a common excipient used in many areas of drug delivery. For example, glycerol is routinely used as emollient, humectant, gel vehicle, plasticiser, taste masker, transdermal patch additive and osmotic agent $[1,2,13]$. Glycerol has a relatively low diffusivity when compared to an aqueous solution $[3,14]$. Thus, the influence of glycerol on drug transport across the respiratory epithelium would be interesting and provide important information regarding the potential effect on bioavailability.
Two solution-based pMDIs with identical aerodynamic properties and aerosol dosimetry, but whose particles contained either drug alone or drug and glycerol at a 1:1 ratio have been developed [15]. Semi-empirically derived equations were used to relate HFA, ethanol, non-volatile components and valve components variables to the aerosol particle size distribution and formulate solution pMDIs with equivalent fine particle doses $(\leqslant 5 \mu \mathrm{m})$ and MMAD. These inhalers generated particles with very different physicochemical properties: while the particles from each inhaler formulation were amorphous, the morphology and thermal properties of the glycerol-containing particles were significantly different than those of the glycerol-free particles.

Following the successful formulation and characterisation of two formulations that had identical aerosol profiles, this article investigates the role of glycerol on the dissolution and absorptive transport of BDP across the respiratory epithelium using an air interface Calu-3 cell-based drug absorption model.

\section{Materials and methods}

\subsection{Materials}

BDP was supplied by Teva Pharmaceuticals (Harlow, UK). Actuators and pMDI valves were provided by Bespak Ltd. (Norfolk, UK). pMDI cans were provided by Presspart Ltd. (Lancashire, UK). Ethanol ( $\geqslant 99.5 \%$ ) was supplied by Sigma-Aldrich (Gillingham, UK) and glycerol $(\geqslant 99 \%)$ provided by Sigma-Aldrich (Gillingham, UK). HFA134a (1,1,1,2-tetrafluoroethane) and HFA 227 (1,1,1,2,3,3,3heptafluoropropane) were supplied by (Mexichem Fluor, Runcorn, UK).

Dulbecco's Modified Eagle's Medium (DMEM, without phenol red and L-glutamine, including sodium bicarbonate and $15 \mathrm{mM}$ HEPES), non-essential amino acids solution (x100), CelLytic ${ }^{\mathrm{TM}} \mathrm{M}$ Cell Lysis (50 mM Tris- $\mathrm{HCl}, \mathrm{pH} 8,150 \mathrm{mM} \mathrm{NaCl}, 1 \% \mathrm{NP}-40,0.5 \%$ sodium deoxycholate, $0.1 \%$ SDS), mammalian protease inhibitor cocktail, HEPES (4-(2-Hydroxyethyl) piperazine-1-ethanesulfonic acid sodium salt) and $( \pm)-\alpha$-Tocopherol (Vitamin E) were purchased from Sigma-Aldrich (Sydney, Australia). Phosphate buffered saline (PBS), Foetal bovine serum (FBS), L-glutamine solution $(200 \mathrm{mM})$, Trypsin-EDTA solution (2.5 g/L trypsin, $0.5 \mathrm{~g} / \mathrm{L}$ EDTA) and Hank's balanced salt solution (HBSS) were obtained from Invitrogen (Sydney, Australia). The Calu-3 cell line (HTB-55) was obtained from American Type Cell Culture Collection (ATCC, Rockville, USA) and was used between passages 35 and 40. Transwell cell culture inserts $\left(0.33 \mathrm{~cm}^{2}\right.$ polyester, $0.4 \mu \mathrm{m}$ pore size $)$ were purchased from Corning Costar (Lowell, MA, USA). Sterile culture plastic-wares were obtained from Sarstedt (Adelaide, Australia). All solvents and chemicals were analytical grade and were supplied by Sigma (Sydney, Australia). Water used throughout the experiment was purified by reverse osmosis (MilliQ Sydney, Australia).

\subsection{Preparation of solution pMDIs with equivalent aerodynamic size distributions}

Formulations exhibiting equivalent MMAD, geometric standard deviations (GSDs) and stage deposition profiles were prepared using methods described previously [15]. The formulations were designed such that evaporation of the volatile components would result in aerosol particles containing either drug alone or drug with $50 \% \mathrm{w} / \mathrm{w}$ glycerol, thus allowing the effect of glycerol on dissolution and transepithelial transport to be studied (Table 1).

These two formulations are referred to 'glycerol-free' and 'glycerol-containing' throughout the remainder of the text. 
Table 1

Formulation and device specification for the glycerol-containing and glycerol-free BDP pressurised metered dose inhalers.

\begin{tabular}{|c|c|c|c|c|}
\hline & \multicolumn{2}{|c|}{$\begin{array}{l}\text { Glycerol- } \\
\text { containing }\end{array}$} & \multicolumn{2}{|c|}{ Glycerol-free } \\
\hline & $\begin{array}{l}\% \mathrm{w} / \\
\mathrm{w}\end{array}$ & $\mathrm{mg}$ & $\begin{array}{l}\% \mathrm{w} / \\
\mathrm{w}\end{array}$ & $\mathrm{mg}$ \\
\hline BDP & 0.35 & 60.6 & 0.43 & 59.3 \\
\hline Ethanol & 15.00 & 2595.0 & 15.00 & 2092.5 \\
\hline Glycerol & 0.35 & 60.6 & 0.0 & 0.0 \\
\hline HFA 134a & 84.3 & 14583.9 & 0.0 & 0.0 \\
\hline HFA 227 & 0.0 & 0.0 & 84.57 & 11798.2 \\
\hline Bespak metering chamber $(\mu \mathrm{l})$ & \multicolumn{2}{|l|}{$63 \mu \mathrm{l}$} & \multicolumn{2}{|l|}{$50 \mu \mathrm{l}$} \\
\hline Bespak actuator orifice diameter ( $\mathrm{mm})$ & \multicolumn{2}{|l|}{0.30} & \multicolumn{2}{|l|}{0.33} \\
\hline
\end{tabular}

\subsection{Cell culture}

Calu-3 cell monolayer was grown in Dulbecco's Modified Eagle's medium: F-12 containing $10 \%(\mathrm{v} / \mathrm{v})$ foetal calf serum, $1 \%(\mathrm{v} / \mathrm{v})$ nonessential amino acid solution and $1 \%(\mathrm{v} / \mathrm{v})$ L-glutamine solution. The cells were seeded on Transwell inserts at a density of $5 \times 10^{5}$ cells $\times \mathrm{cm}^{-2}$ and maintained in $95 \% \mathrm{rH}$ air supplemented with $5 \% \mathrm{CO}_{2}$ at $37^{\circ} \mathrm{C}$. After $24 \mathrm{~h}$ in culture, the medium covering the cells in the apical chamber was removed and the cells were allowed to differentiate under the air interface condition. Drug permeability studies were conducted on cell layers between 12 and 14 days after seeding. This period was previously determined as optimal by Haghi et al. $[6,16]$.

The transepithelial electrical resistance (TEER) of the Calu-3 monolayer was evaluated before and after the transport study to confirm that cell layer integrity was maintained over the course of the experiment. To enable TEER measurements, $100 \mu \mathrm{L}$ prewarmed medium was added to apical chamber and TEER was measured with a voltohmmeter (EVOM with STX-2 chopstick electrodes, World Precision Instruments, FL, USA). TEER measurements at the end of each experiment showed no significant differences when compared to pre-experiment values (ANOVA $p<0.05$ ).

\subsection{In vitro cell deposition studies}

Deposition of particles generated by each formulation onto the cell layers and measurement of transepithelial BDP flux was conducted using methods adapted from Grainger et al. [10,17]. In this method, the twin stage impinger (TSI) (Radleys, Essex, UK) was modified to accommodate a Transwell insert in the lower chamber. The assembled TSI was then connected to a Rotary vein pump (GAST, Michigan, USA). The pMDIs containing the BDP formulations were connected to the TSI and the flow rate adjusted to $60 \mathrm{~L} \times \mathrm{min}^{-1}$ using a flow metre (Model 4040, TSI Model Instruments, Germany). The pMDIs were then actuated a set number of times (ranging from 2 to 10 shots; to allow the deposition of equivalent total drug mass or total particle number on the cell layer). After particle deposition, the Transwell was removed from the TSI, the outer surface cleaned of the adhering particles and transferred into a well containing $300 \mu \mathrm{L}$ modified buffer (HBSS supplemented with HEPES (1\% v/v), vitamin E (1\% v/v) and ethanol $97 \%$ $(1 \% \mathrm{v} / \mathrm{v})$ at $\mathrm{pH} 7.4)$ to measure the BDP transfer. The cells were pre-equilibrated in the modified buffer for $1 \mathrm{~h}$ prior to particle deposition. At each time point, the cells were moved to a new well containing fresh medium. At the end of 4-h experiment, the medium from the wells was collected into vials and the wells were rinsed with $300 \mu \mathrm{L}$ of ether to recover any $\mathrm{BDP} / \mathrm{BMP}$ adhered to the plastic. A fluticasone internal standard for high performance liquid chromatography mass spectrometry (HPLC-MS) and ether used for rinsing were also added to the samples. After $4 \mathrm{~h}$ of experiment, the surface of the monolayer was washed and the sample collected for analysis of drug content. The cell layer was then harvested for analysis of the drug inside the cells. The method has been described previously by Haghi et al. $[3,18]$. Briefly, the cells were harvested using trypsin EDTA solution and lysed using Cell lytic and protease inhibitor cocktails. The samples were vortexed and left in freezer at $-70^{\circ} \mathrm{C}$. Once the aqueous phase was frozen, the ether phase was removed and evaporated. The samples were reconstituted in mobile phase and analysed with HPLC-MS.

The amount of BDP deposited on the cell layers was verified in preliminary studies by depositing particles onto cell-free Transwells and immediately washing to recover the BDP. In the transport experiments, the cumulative amount of BDP transported across the cell layer was added to the amount of BDP within and on the surface of the cell layer. Thus, the total amount of BDP deposited and the percentage BDP transported were calculated (metabolism of BDP to its major metabolite BMP occurs with 1:1 stochiometry and was also accounted for and used to calculate the 'total' amount of BDP deposited and 'total drug' transported). The total drug (BDP + BMP) recovered during experiments was consistent with the amount of BDP deposited onto cell-free Transwells in the dose-finding studies.

\subsection{High performance liquid chromatography mass spectrometry (HPLC-MS)}

An HPLC-MS method was used to determine the concentration of BDP and the primary metabolite from in vitro cell samples. Briefly, a Thermo Scientific TSQ quantum access max system was utilised comprising of a Transcend 600 series TLX1 (Thermo Scientific Ltd., Australia).

A $75 \mathrm{~mm} \times 2.1 \mathrm{~mm}(2.7 \mu \mathrm{m}$ Fused-Core particle platform $)$ Supelco Ascentis Express C18 column was used for separation. After the ether extraction process described previously, the dry analytes were reconstituted with $200 \mu \mathrm{L}$ mobile phase consisting of $(80: 20 \%, v / v)$ methanol: $10 \mathrm{mM}$ aqueous ammonium acetate solution and the internal standard (fluticasone) were added to each sample. Each sample was injected ( $20 \mu \mathrm{L}$ injection volume) onto the column, operating at a flow rate of $0.2 \mathrm{~mL} \times \mathrm{min}^{-1}$.

The mass spectrometer was operated in a positive electrospray ionisation mode, with a $5 \mathrm{kV}$ capillary, a $17 \mathrm{~V}$ cone, Vaporizer Temperature and Ion Transfer Tube Temperature of $382{ }^{\circ} \mathrm{C}$ and $250{ }^{\circ} \mathrm{C}$, respectively. The selective reaction-monitoring (SRM) mode was used for mass spectrometry detection. Parent ion channels of 501,521 and $465 \mathrm{~m} / \mathrm{z}$ and daughter ion channels of 313, 319 and $279 \mathrm{~m} / \mathrm{z}$ were used for fluticasone, BDP and BMP, respectively.

\subsection{Statistical analysis}

Data were analysed for statistical significance using the SPSS Statistics 17.0 software package (SPSS Inc., Chicago, IL, USA). One-way ANOVA (with Tukey's post hoc analysis) was utilised to test for significance. Data are represented as mean \pm SD of minimum five independent experiments. Differences were considered significant when $p<0.05$.

\section{Results and discussion}

The surface of the airway epithelium is a complex pseudo-stratified structure made up of a number of differentiated cell types and a surface lining fluid containing mucus and phospholipids. The surface area of the epithelium is large, and deposition of unagglomerated inhaled drug particles is likely to be a discrete event. In the tracheobronchial region, the volume of the mucosal lining liquid is low and the layer of mucus is of an equivalent thickness to the 
diameter of particles that typically deposit in that region $[3,19]$. It may therefore be envisaged that when a particle deposits, the surrounding mucus rapidly becomes saturated with drug and passive absorptive transport is dependent on the properties of the drug and epithelium (i.e. partition and diffusivity of drug via the transcellular and paracellular routes). However, this process becomes more complicated when delivering a semi-solid particle such as the glycerol formulation, which contains BDP glycerol at a 1:1 mass ratio as the effect of the excipient, must also be considered.

This experiment was designed, so that the aerodynamic size distribution of the BDP particles from the glycerol-free and glycerol-containing formulations was equivalent, allowing for a direct comparison of the influence of glycerol on transepithelial drug transport. The MMAD of the glycerol-free and glycerol-containing formulations, measured via ACI stage deposition data, was $2.4 \pm 0.1 \mu \mathrm{m}$ and $2.5 \pm 0.2 \mu \mathrm{m}$, respectively $(n=3)$. No significant difference in stage-by-stage deposition or fine particle fraction/ mass was observed between the two formulations used $[8,15]$. It is important to note, however, that for the mass of drug emitted by both formulations to be similar, the number of particles (and formulation mass, including excipient) would be different. Since the number of particles or total drug mass on the epithelial surface may affect drug transport, matched doses of each were incorporated into the study design.

\subsection{Dosimetry: particle number versus mass of BDP delivered}

The number of actuations into the TSI was optimised to deposit equivalent particle number or equivalent BDP mass onto the Transwell or Calu-3 cell layer. It is important to note that the exact number of particles can only be estimated, as the actual distribution of particles within the respective particle size classes is not precisely known. However, the particle size distribution MMAD and GSD for each formulation were not significantly different making comparison by mass possible [15]. After the evaporation of the volatile HFA and ethanol components, both the glycerol-free and glycerol-containing particles had similar size distributions and delivered equivalent BDP mass per actuation. Thus, aerodynamic deposition will result in twice the number of glycerol-containing particles compared to the glycerol-free formulation since glycerol accounts for $50 \% \mathrm{w} / \mathrm{w}$ of the total particle mass. This concept is illustrated in (Fig. 1).

The influence of glycerol on the disposition of BDP was investigated by delivering an equivalent mass of BDP from each formulation $(2215 \mathrm{ng} \pm 271 \mathrm{ng}$ of BDP from the glycerol-free formulation compared to $1909 \mathrm{ng} \pm 249 \mathrm{ng}$ of BDP from the glycerol-containing formulation). The effect of depositing equivalent particle numbers was investigated by delivering $2215 \mathrm{ng} \pm 271 \mathrm{ng}$ BDP from the glycerol-free formulation compared to $826 \mathrm{ng} \pm 138 \mathrm{ng}$ BDP from the glycerol-containing formulation. In addition, as a control for the effect of mass of BDP deposited (i.e. 1000 ng and 2000 ng) versus the formulation effect, a lower deposition of glycerol-free formulation was also performed (1024 $\mathrm{ng} \pm 142 \mathrm{ng}$ BDP).

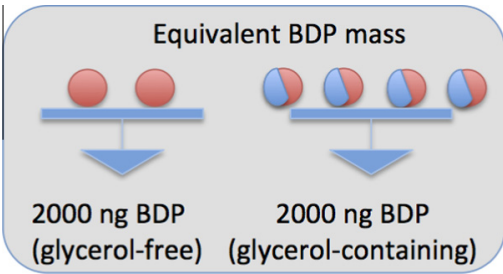

The density of particles on the epithelial cell layer surface was estimated to address concerns regarding particle overload. Taking the 'worst case' scenario by considering the surface area of the cell monolayer $\left(0.33 \mathrm{~cm}^{2}\right)$ and assuming a particle diameter of $2.5 \mu \mathrm{m}$ (based on the MMAD), the lowest theoretical particle density of $1260 \mathrm{~kg} \mathrm{~m}^{3}$ (i.e. that of glycerol [8,13]) and a deposited mass of $5 \mu \mathrm{g}$ (i.e. twice that of the maximum BDP mass deposited), the coverage of the cell surface would be $\sim 7 \%$. Although this calculation is based on spherical particle geometries and their projected surface area, it is clear that none of the doses utilised would overload the cells or layer particles upon each other.

\subsection{Kinetics of BDP transport across Calu-3 cells}

The influence of depositing BDP (1000 ng or $2000 \mathrm{ng}$ in glycerol-free or glycerol-containing particles) on the mass or percentage transport of BDP across the Calu-3 cell line was studied.

\subsubsection{BDP transport after deposition of glycerol-free particles}

The glycerol-free formulation delivered BDP across the cell layer in proportion to the mass of drug deposited onto the cells, with the rate of transport remaining linear irrespective of the mass of BDP deposited (i.e. constant flux, zero-order kinetics). Thus, $35.5 \pm 5.8 \mathrm{ng}$ and $101.6 \pm 31.5 \mathrm{ng}$ of BDP was transported over $2 \mathrm{~h}$ following the deposition of $\sim 1000$ and $\sim 2000 \mathrm{ng}$ BDP, respectively. These results are consistent with particles being depositing discretely at the epithelia and immediately saturating the local mucus-rich cell lining fluid. The rate of drug transport was directly proportional to the number of particles deposited discreetly across the epithelia, i.e. the percentage of BDP transported was the same irrespective of the amount of BDP deposited (3.3-4.1\% over $2 \mathrm{~h}$; Fig. 2).

\subsubsection{BDP transport after deposition of glycerol-containing particles}

In contrast to the non-glycerol formulation, the transport of BDP from the glycerol-containing formulation was independent of the mass of BDP deposited (Fig. 2). Approximately $50 \mathrm{ng}$ of BDP was transported across the cell layer $(51.2 \pm 3.1 \mathrm{ng}$ and $58.0 \pm 10.0 \mathrm{ng}$ ) after deposition of particles containing $\sim 1000 \mathrm{ng}$ and $2000 \mathrm{ng}$ BDP, respectively. Interestingly, as with the glycerol-free formulation, the transport rate was linear (i.e. constant flux suggesting zero-order kinetics); however, the mechanism of drug dissolution/release at the epithelial surface was clearly different. Since the mass transport of BDP was independent of the deposited dose, the percentage transported varied significantly $4.9 \pm 0.1 \%$ and $2.2 \pm 0.0 \%$ in $2 \mathrm{~h}$ following deposition of glycerolcontaining particles delivering $\sim 1000 \mathrm{ng}$ and $\sim 2000 \mathrm{ng}$ BDP, respectively. This constant mass transfer of BDP from glycerol-containing particles suggests a significant effect of glycerol upon the BDP availability at the cellular level.

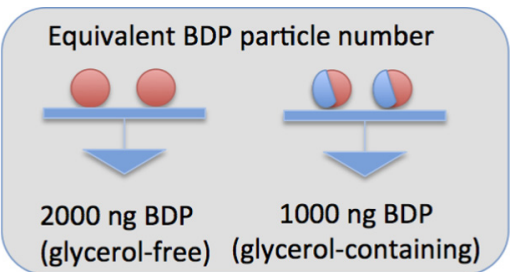

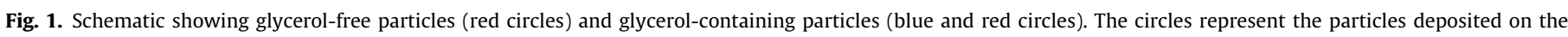

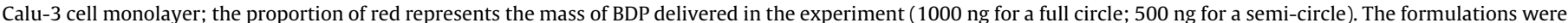

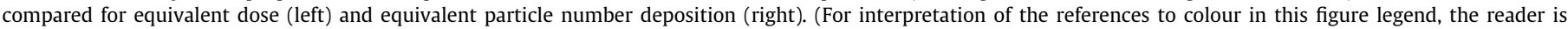
referred to the web version of this article.) 

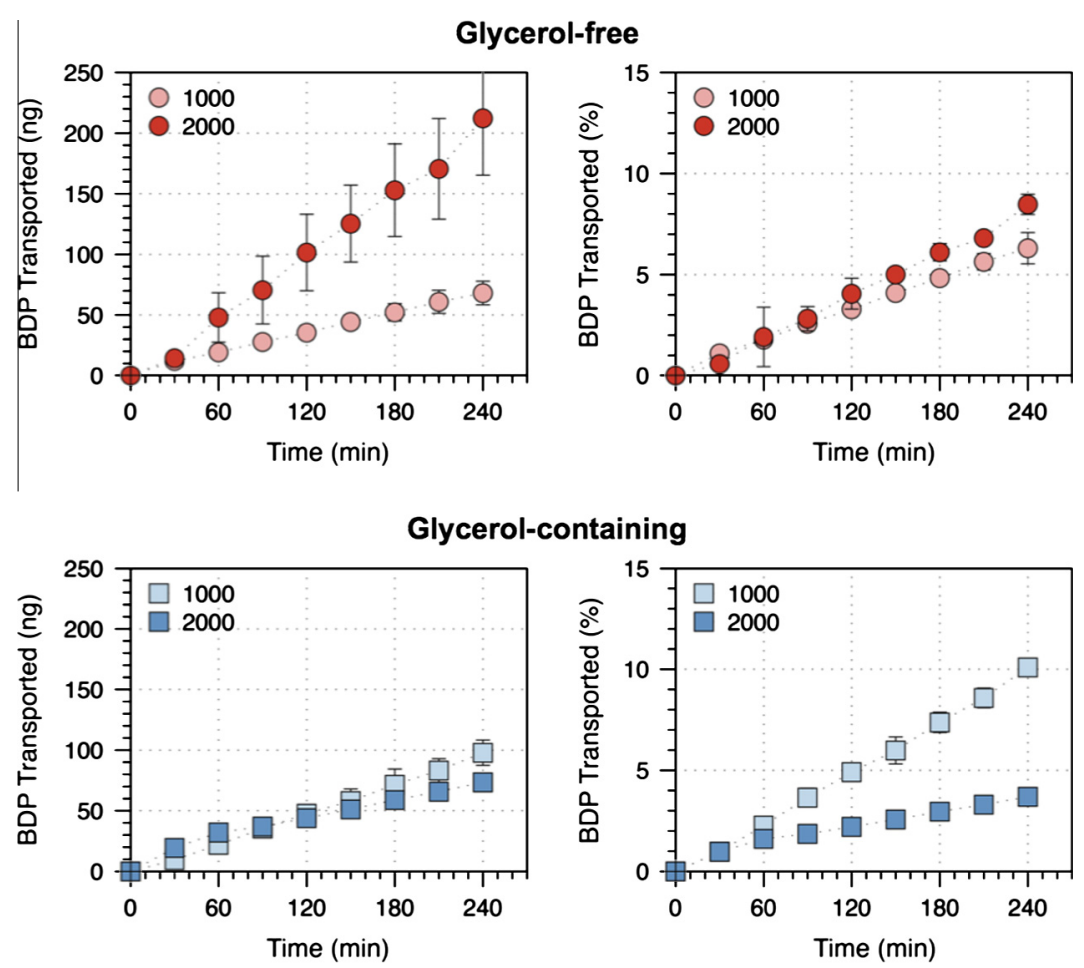

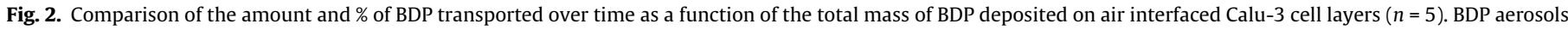

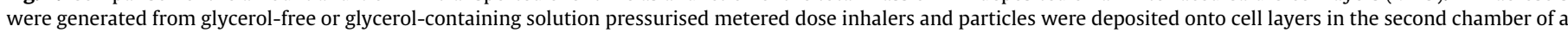
twin stage impinger. (For interpretation of the references to colour in this figure legend, the reader is referred to the web version of this article.)

\subsection{Glycerol-containing versus glycerol-free formulations}

TEER measurements at the end of each experiment showed no significant differences when compared to pre-experiment values (ANOVA $p<0.05$ ).

The transport kinetics of BDP across the Calu-3 cells were reanalysed to compare BDP transport from glycerol-free and glycerol-containing particles in terms of mass and percentage transport after deposition of an equivalent mass of BDP ( 2000 ng) or an equivalent number of particles from each formulation deposited onto the cells (Fig. 3).

The transport rate of BDP was higher in the absence of glycerol in the particles (Fig. 3A); this was clearly seen when presented as the percentage transport data with $4.1 \pm 0.8 \%$ of BDP from the glycerol-free formulation transported to the basal compartment over $2 \mathrm{~h}$ compared to $2.2 \pm 0.0 \%$ from the glycerol formulation (Fig. 3B). The mass and percentage of BDP transported when an equivalent number of particles was deposited on the cells ( $\sim 2000 \mathrm{ng}$ BDP in the glycerol-free particles compared with $\sim 1000 \mathrm{ng}$ BDP in the glycerol-containing particles) revealed a higher transport rate over $2 \mathrm{~h}$ for BDP from the glycerol-free formulation compared to the glycerol-containing formulation (Fig. 3C). Incidentally, the percentage BDP transport from both these formulations was similar (Fig. 3D; $4.06 \pm 0.77 \%$ and $4.91 \pm 0.10 \%$ for BDP from non-glycerol and glycerol formulations, respectively). Interestingly, these profiles were derived from the equivalent number of glycerol-free particles delivering double the mass of BDP compared to the glycerol-containing particles.

\subsection{Metabolism of BDP to BMP and 'total drug' transport}

In addition to the transport of BDP, it is important to consider metabolism by the respiratory epithelial cells. BDP is metabolised to beclomethasone 17-monopropionate (17-BMP), beclomethasone 21-monopropionate (21-BMP) and beclometha- sone (BOH). Previous studies have shown that the relative binding affinity of 17-BMP for the cytoplasmic glucocorticoid receptor of human lung is $\sim 25$ times greater than that of the parent drug BDP $[3,20]$, whereas $21-B M P$ is practically inactive. The amount of 17-BMP generated and transported in the experiments was proportional to the levels of BDP transported across the cells (Fig. 4), suggesting that the differences in metabolism/transport of BDP in the presence or absence of glycerol reflect the availability of BDP from these formulations (i.e. the action of glycerol in reducing transepithelial flux of BDP also results in decreased drug exposure to metabolising enzymes in the cell cytoplasm). This is consistent with the amount of BDP within the cells at the end of the experiments: cells exposed to glycerol-free formulation contained $50.7 \mathrm{ng} \pm 28.8 \mathrm{ng}$ and $112.3 \mathrm{ng} \pm 26.6 \mathrm{ng}$ after the deposition of $1000 \mathrm{ng}$ and $2000 \mathrm{ng}$ BDP, respectively, whereas there was no significant difference in the amount of BDP recovered from the cells was observed for the glycerol formulation (33.9 ng $\pm 13.3 \mathrm{ng}$ and $28.8 \mathrm{ng} \pm 7.1 \mathrm{ng}$ for $1000 \mathrm{ng}$ and $2000 \mathrm{ng}$ BDP deposited, respectively).

Interestingly, there was an appreciable lag of $\geqslant 60 \mathrm{~min}$, before BMP appeared in the receiver chamber of the Transwell diffusion apparatus (i.e. on the contralateral side of the Calu-3 cell layer after BDP deposition to the apical surface). This may simply reflect the time taken for appreciable levels of 17-BMP to be generated, accumulate and efflux from the cells. However, BMP appearance in the basal compartment may depend, in part, on the binding affinity and saturation of the glucocorticoid receptor in the cells (an effect that would not be seen with BDP since the pro-drug has a much lower receptor affinity than 17-BMP $[11,19])$. It is also interesting to speculate whether glycerol may interact with BDP sterically to hinder metabolism by reducing the access of esterase enzymes to the ester carbonyl in the BDP molecule [10,11,21].

When 'total drug' transport is considered (i.e. combining 17-BMP and the BDP data), the trends discussed for BDP transport were mirrored (Fig. 5). A mass of $465.0 \mathrm{ng} \pm 95.1 \mathrm{ng}$ of the total 

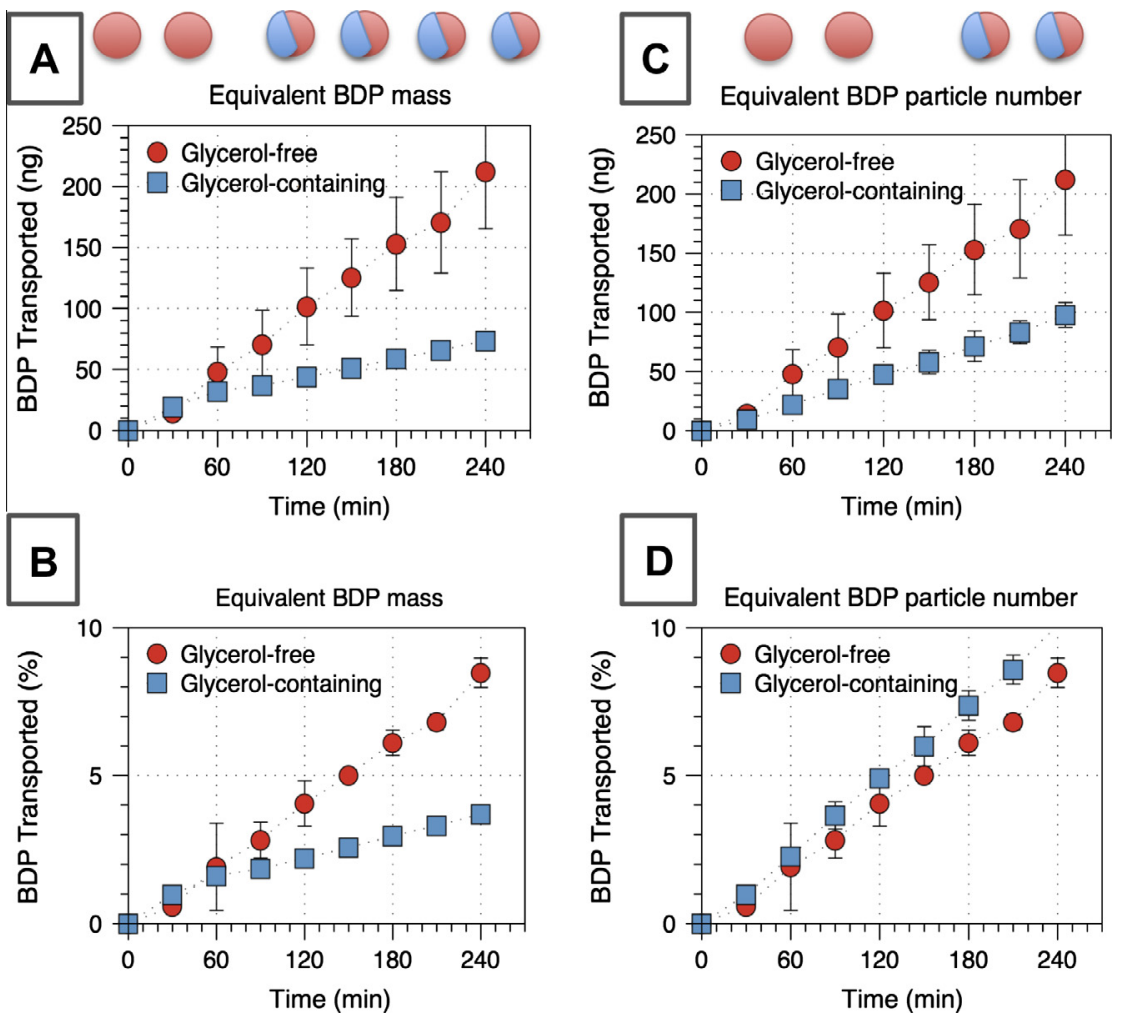

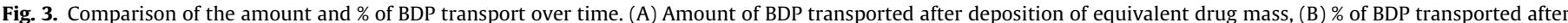

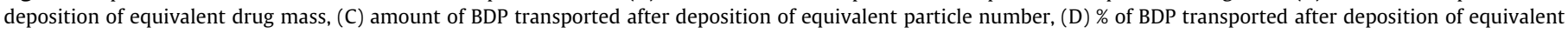
particle number $(n=5)$. (For interpretation of the references to colour in this figure legend, the reader is referred to the web version of this article.)
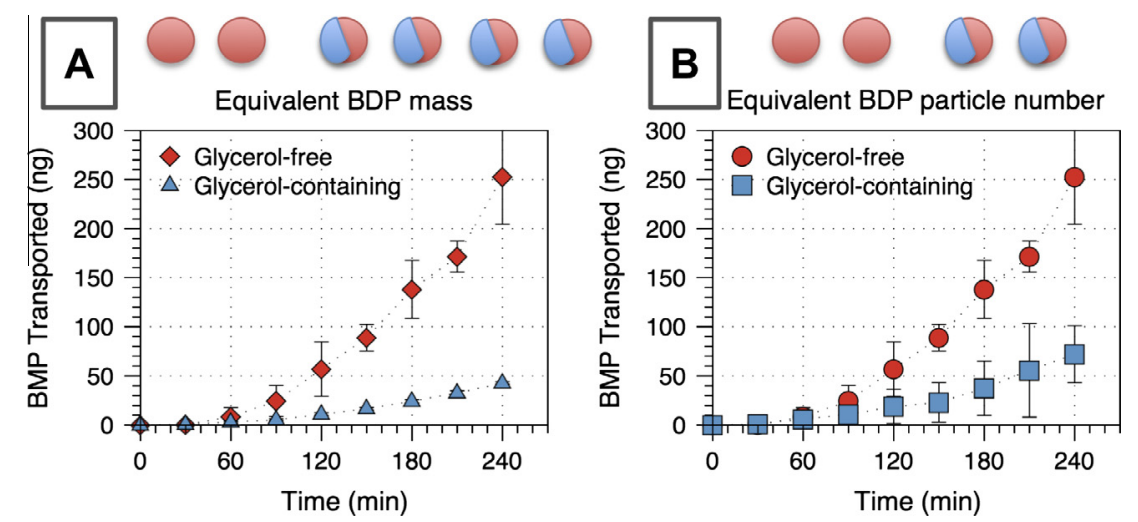

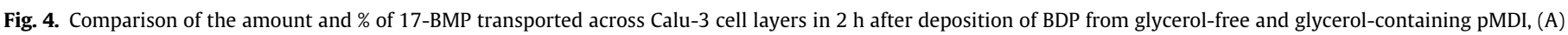

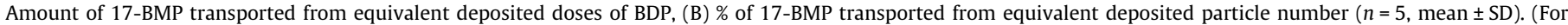
interpretation of the references to colour in this figure legend, the reader is referred to the web version of this article.)

drug $(20.99 \pm 4.29 \%)$ was transported through the cell layer in the glycerol-free formulation compared to $116.17 \mathrm{ng} \pm 3.07 \mathrm{ng}$ total drug $(6.07 \pm 0.16 \%)$ for the glycerol-containing formulation over the duration of the experiment (Fig. 5A and B). Similarly, total drug transport after deposition of equivalent particle number (Fig. 5C and D) showed that total drug transport from the glycerol-free particles was $465.01 \pm 95.12 \mathrm{ng}(20.99 \pm 4.29 \%$ of total deposition $)$ compared with $169.83 \pm 39.52 \mathrm{ng}(20.56 \pm 4.78 \%)$ from the glycerol-containing formulation. As with the BDP and BMP transport data (in Sections 3.2 and 3.3), it is clear that the total amount of drug transported across the epithelia (BDP and BMP) is dependent on the formulation deposited.

\subsection{Effect of glycerol on BDP availability}

In conventional gel-based formulations, glycerol is often incorporated in an aqueous solution at concentrations ranging from $0.5 \%$ to $50 \% \mathrm{w} / \mathrm{v}[10,13]$; however, in the formulation studied here, the dried aerosol particles would be solid or semi-solid with a $1: 1$ mass ratio of amorphous BDP and glycerol. The presence of glycerol appeared to affect the release of drug from the deposited particle, with resultant consequences for the rate of transport across the epithelium. We have previously reported that the presence of glycerol in the aerosolised droplets affected the physicochemical properties of the particles generated after evaporation of volatile 

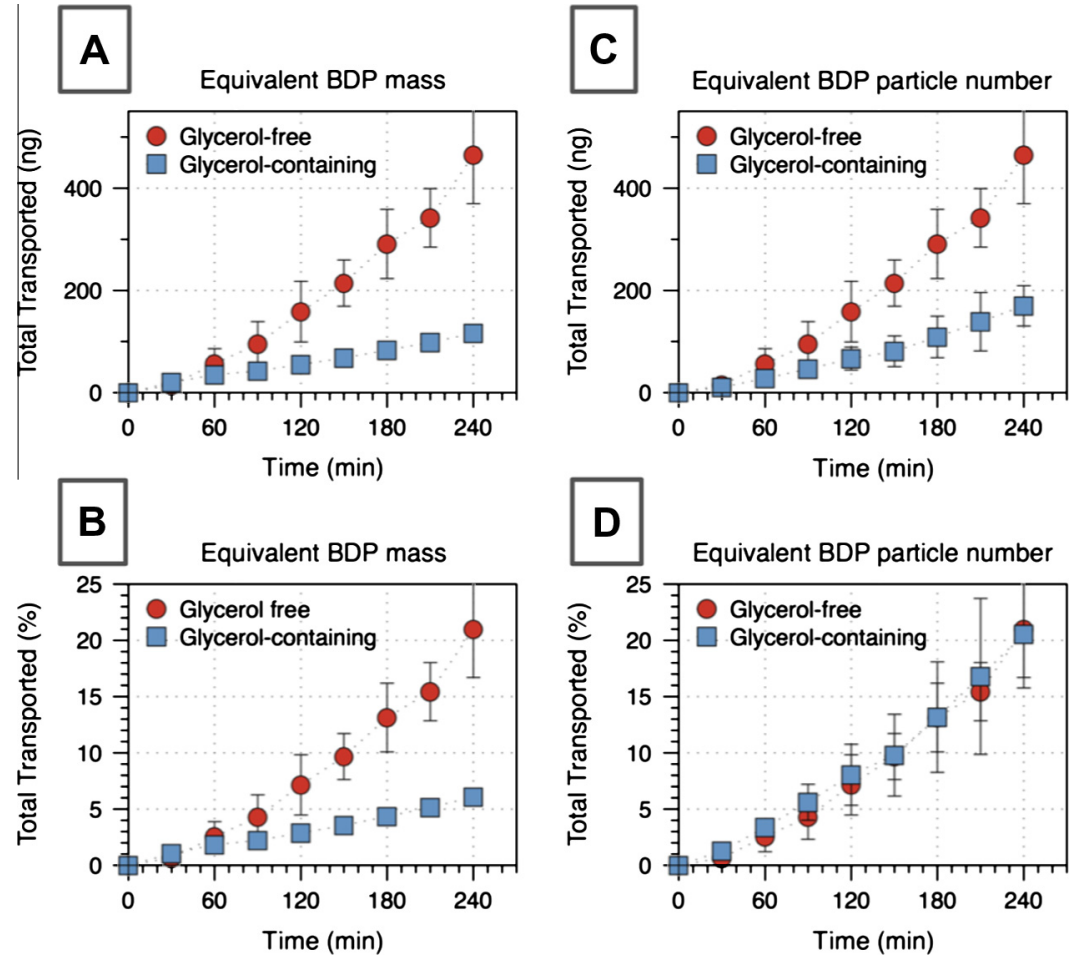

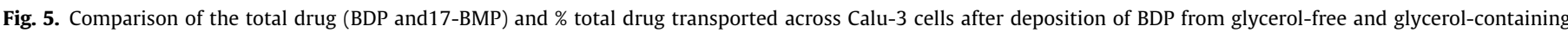

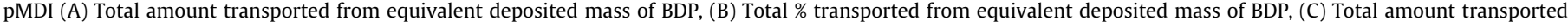

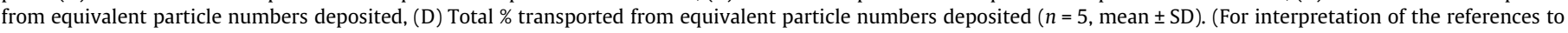
colour in this figure legend, the reader is referred to the web version of this article.)

components and the release of BDP across a non-cellular membrane in a Franz diffusion cell $[10,15]$.

Glycerol is able to hydrogen bond intra-molecularly and intermolecular with BDP and water. Molecular dynamic stimulations and infrared spectroscopy have provided evidence of glycerol and water hydrogen bonding patterns [22]. It has been shown by infrared spectroscopy that increasing glycerol concentration causes a growth in the first hydration shell since more bulk water molecules are required for the solvation of glycerol. The surface of the epithelial cell layers used here provides a fluid restricted, glycoprotein rich environment, which is representative of the surface of the tracheobronchial region of the lung. The amount of fluid on the surface of air interfaced Calu-3 cells has been previously estimated at around $3.44 \mu \mathrm{L}$ over a $0.33 \mathrm{~cm}^{2}$ area [17]. The amounts of drug/glycerol deposited in this study may have depleted the bulk water pool to a point there are insufficient free water molecules to transfer to the first hydration shell with the result that water is no longer available to solvate the glycerol molecules and the rate of BDP dissolution and diffusion is diminished.

It has also been shown that as the glycerol concentration increases, water molecules in the first hydration shell are more likely to be found around glycerol $\mathrm{OH}$ groups. This implies that the $\mathrm{CH}$ groups of BDP are surrounded by glycerol in an arrangement known as alkyl backbone clustering. At higher concentrations of glycerol, water molecules concentrate around the polar groups of glycerol causing the alkyl groups of glycerol to self-associate [22] and glycerol-glycerol hydrogen bonds become dominant. This may result in the formation of a gel layer consisting of structured Water-Glycerol-BDP, the properties of which dictate the kinetics of drug release.

In general, the results of this study are in good agreement with previous in vitro [10] and ex vivo [11] studies on commercial products QVAR and Sanasthmax. However, in the earlier investigations, the specific effect of glycerol could not be delineated from other variables in the particle (especially size). In this study, the aerodynamic diameters of the glycerol and non-glycerol formulation were matched. Although clear differences in in vitro epithelial transport were observed, the availability of BDP from a glycerol-rich gel in a fluid restricted environment is the likely reason for the observations as this effect was also seen in the cell free (Franz diffusion apparatus) dissolution studies. Future studies should investigate the influence of glycerol on particle dissolution and drug diffusion at a molecular level and investigate the absorption of BDP in vivo after delivery of aerosols generated from these two formulations.

\section{Conclusions}

Two formulations with identical MMADs, containing BDP alone or $\mathrm{BDP} /$ glycerol in a 1:1 ratio, were deposited onto air interfaced Calu-3 epithelial cell layers. Significant differences in the rate of drug transport were found, with BDP delivered by the glycerol-free formulation transferring more rapidly across the cell layers. Interestingly, there was a concomitant increase in the extent of metabolism compared to the glycerol-containing formulation. It was concluded that despite the aerodynamic equivalence of the two formulated products, in this model, the presence of glycerol in the deposited particles retarded the availability of BDP for transport across in vitro respiratory epithelial cells. Such observations would suggest that while the two formulations are bio-equivalent in terms of respiratory deposition their bio-equivalency in terms of drug absorption might differ in vivo.

\section{References}

[1] P. Barnes, Efficacy of inhaled corticosteroids in asthma, J. Allergy Clin. Immunol. (1998).

[2] D.M. Lang, New asthma guidelines emphasize control, regular monitoring, Cleveland Clin. J. Med. 75 (9) (2008) 641-653. 
[3] E. Derom, R.A. Pauwels, Pharmacokinetic and pharmacodynamic properties of inhaled beclometasone dipropionate delivered via hydrofluoroalkanecontaining devices, Clin. Pharmacokinet. 44 (8) (2005) 815-836.

[4] P. Rogueda, A. Lallement, D. Traini, I. Iliev, P. Young, Twenty years of HFA pMDI patents: facts and perspectives, J. Pharm. Pharmacol. 64 (9) (2012) 1209-1216.

[5] J. Bell, S. Newman, The rejuvenated pressurised metered dose inhaler, Expert Opin. Drug. Deliv. 4 (3) (2007) 215-234.

[6] D. Ganderton, D.A. Lewis, R. Davies, S. Matsusaka, G.R. Brambilla, T. Church Modulite $^{\circledR}$ : a means of designing the aerosols generated by pressurized metered dose inhalers, Respir. Med. 96 (2002) S3-S8.

[7] M. Dolovich, Imaging drug delivery and drug responses in the lung, Proc. Am. Thoracic Soc. 1 (4) (2004) 329-337.

[8] A. Woodcock, D. Acerbi, G. Poli, Modulite technology: pharmacodynamic and pharmacokinetic implications, Respir. Med. 96 (2002) S9-S15.

[9] D. Acerbi, G.R. Brambilla, I. Kottakis, Advances in asthma and COPD management: delivering CFC-free inhaled therapy using Modulite $^{\circledR}$ technology, Pulm. Pharmacol. Therapy 20 (3) (2007) 290-303.

[10] C.I. Grainger, M. Saunders, F. Buttini, R. Telford, L.L. Merolla, G.P. Martin, S.A Jones, B. Forbes, Critical characteristics for corticosteroid solution metered dose inhaler bioequivalence, Mol. Pharm. 9 (3) (2012) 563-569.

[11] M. Freiwald, A. Valotis, A. Kirschbaum, M. McClellan, T. Mürdter, P. Fritz, G. Friedel, M. Thomas, P. Högger, Monitoring the initial pulmonary absorption of two different beclomethasone dipropionate aerosols employing a human lung reperfusion model, Respir. Res. 6 (2005) 21.

[12] W.P. Adams, R.C. Ahrens, M.-L. Chen, D. Christopher, B.A. Chowdhury, D.P. Conner, R. Dalby, K. Fitzgerald, L. Hendeles, A.J. Hickey, G. Hochhaus, L.B Laube, P. Lucas, S.L. Lee, S. Lyapustina, B. Li, D. O'Connor, N. Parikh, D.A Parkins, P. Peri, G.R. Pitcairn, M. Riebe, P. Roy, T. Shah, G.J.P. Singh, S. Suarez Sharp, J.D. Suman, M. Weda, J. Woodcock, L. Yu, Demonstrating bioequivalence of locally acting orally inhaled drug products (OIPs): workshop summary report, J. Aerosol Med. Pulm. Drug Deliv. 23 (1) (2010) 1-29.

[13] F.A. Alvarez-Nunez, C. Medina, Glycerin, in: R.C. Rowe, P.J. Sheskey, M.E. Quinn (Eds.), Handbook of Pharmaceutical Excipients, sixth ed. Pharmaceutical Press, London, 2009, pp. 283-286.
[14] J. Kristl, S. Pecar, J. Korbarsmid, F. Demsar, M. Schara, Drug diffusion - a field gradient electron-paramagnetic resonance study, Drug Dev. Ind. Pharm. 15 (9) (1989) 1423-1440.

[15] D.A. Lewis, P. Young, F. Buttini, T. Church, P. Colombo, B. Forbes, M. Haghi, R. Johnson, H. O'Shea, R.O. Salama, Bioequivalence of inhalable beclamethasone dipropionate pressurised metered dose inhalers and the influence of nonvolatile excipients 1 : design and characterisation of aerodynamically equivalent formulations with and without glycerol, Eur. J. Pharm. Biopharm. (submitted for publication)

[16] M. Haghi, P. Young, D. Traini, R. Jaiswal, J. Gong, M. Bebawy, Time- and passage-dependent characteristics of a Calu-3 respiratory epithelial cell model, Drug Dev. Ind. Pharm. 36 (10) (2010) 1207-1214.

[17] C.I. Grainger, L.L. Greenwell, G.P. Martin, B. Forbes, The permeability of large molecular weight solutes following particle delivery to air-interfaced cells that model the respiratory mucosa, Eur. J. Pharm. Biopharm. 71 (2) (2009) 318324.

[18] M. Haghi, R.O. Salama, D. Traini, M. Bebawy, P. Young, Modification of disodium cromoglycate passage across lung epithelium in vitro via incorporation into polymeric microparticles, AAPS J. 14 (1) (2012) 79-86.

[19] J.S. Patton, P.R. byron, Inhaling medicines: delivering drugs to the body through the lungs, Nat. Rev. Drug Discov. 6 (1) (2007) 67-74.

[20] P.T. Daley-Yates, A.C. Price, J.R. Sisson, A. Pereira, N. Dallow, Beclomethasone dipropionate: absolute bioavailability, pharmacokinetics and metabolism following intravenous, oral, intranasal and inhaled administration in man, Br. J. Clin. Pharmacol. 51 (5) (2001) 400-409.

[21] F. Mehrnejad, M.M. Ghahremanpour, M. Khadem-Maaref, F. Doustdar, Effects of osmolytes on the helical conformation of model peptide: molecular dynamics simulation, J. Chem. Phys. 134 (3) (2011) 035104.

[22] J.L. Dashnau, N.V. Nucci, K.A. Sharp, J.M. Vanderkooi, Hydrogen bonding and the cryoprotective properties of glycerol/water mixtures, J. Phys. Chem. B 110 (27) (2006) 13670-13677. 